\title{
Electrodes Modified with Poly(3,4-Ethylenedioxythiophene) Doped with Sulfonated Polyarylethersulfones: Towards New Conducting Polymers
}

\author{
V. Sabatini ${ }^{\mathrm{a}, \mathrm{b}}$, V. Pifferi ${ }^{\mathrm{a}, \mathrm{b}}$, S. Checchia ${ }^{\mathrm{c}}$, S. Rebeccani ${ }^{\mathrm{a}, \mathrm{b}}$, H. Farina ${ }^{\mathrm{a}, \mathrm{b}}$, Marco A. \\ Ortenzi $^{\mathrm{a}, \mathrm{b}}$ and L. Falciola ${ }^{\mathrm{a}, \mathrm{b}}$ \\ ${ }^{a}$ Department of Chemistry, Università degli Studi di Milano, Via Golgi 19, 20133 Milano, Italy \\ ${ }^{b}$ Laboratory of Materials \&Polymers (LaMPo), Università degli Studi di Milano, Via Golgi 19, 20133 Milano, Italy \\ ${ }^{c}$ ESRF- The European Synchrotron, 71 Avenue des Martyrs, Grenoble 38000, France
}

\begin{abstract}
To overcome the well-known technical problems of Poly(3,4-Ethylenedioxythiophene) (PEDOT), i.e. difficult processability and patterning, due to its poor solubility in common organic and inorganic solvents, PEDOTs characterized by a full miscibility in the reaction solvents used, were successfully synthesized by a direct oxidative polycondensation reaction between Ethylenedioxythiophene (EDOT) and an oxidant species, i.e. ferric sulfate, in four organic reaction solvents: N, N-dimethylformamide (DMF), dimethylacetamide (DMAc), dimethyl sulfoxide (DMSO) and N-methyl-2-pyrrolidone (NMP).

The oxidative polycondensation of EDOT in the presence of Sulfonated Polyarylethersulfone (SPAES) as doping agent characterized by three increasing degree of sulfonation (DS), i.e. $0.5,0.75$ and 1.0 (meq $\mathrm{SO}_{3}^{-} \cdot \mathrm{g}^{-1}$ of polymer), was performed for the first time, leading to a new conducting material: PEDOT_SPAES. PEDOT_SPAES can be easily processed and casted onto the surface of glassy carbon electrodes, reaching better electrochemical performances with respect to the precursors.

PEDOT_SPAES chemical structure was investigated via wide-angle scattering (WAXS): comparing WAXS spectra of unmodified PEDOT and commercial PEDOT doped with 2-Naphthalenesulfonic acid, having crystalline structures, with the spectra of PEDOT_SPAESs, that are characterized by amorphous structures, it is possible to assess that for the first time PEDOT-based materials doped with SPAES were synthesized.
\end{abstract}

Keywords: conducting polymer, Poly(3,4-Ethylenedioxythiophene), oxidative polycondensation, Sulfonated Polyarylethersulfone, modified electrode, WAXS.

PACS: $82.35 . \mathrm{Cd}$

\section{INTRODUCTION}

In the last decade, Polythiophene and its derivates have been at the center of considerable scientific interest for their attractive physico-chemical properties and conductive performances [1]. Among Polythiophene derivates, Poly(3,4-Ethylenedioxythiophene) (PEDOT) is one of the most successful commercial conducting polymer due to its low bandgap, excellent environmental stability, high electrical conductivity and transparency when in the form of thin oxidized film [2]. Since the first synthesis of PEDOT in 1989, described in Roncali review [3], several studies on this polymer have been carried out [4] and many applications have been reported for solid electrolytic capacitors, anti-electrostatic agents, electrochemical electrodes, transparent electrodes in light emitting diodes, and under layers for the metallization of printed circuit boards [5].

Numerous researches have been made on the synthesis of PEDOT by electrochemical [6] and chemical polymerization [7] of 3,4-Ethylenedioxythiophene (EDOT) monomer, that has been recognized as more suitable for mass production than other possible thiophene-based monomers [8]. In 2005, Lei and others [9] suggested a new chemical route for PEDOT polymerization: the synthesis is performed by high-concentration water-based emulsion polymerization of EDOT in the presence of 2-Naphthalenesulfonic acid as doping agent and ferric sulfate as oxidant 
species; during the polymerization, PEDOT precipitates as a dark-blue powder insoluble in the reaction solvent, i.e. water. The electrical conductivity of PEDOT obtained using this polymerization technique resulted to be around 160 $\mathrm{S} \cdot \mathrm{cm}^{-1}$, an excellent value for an organic conducting polymer.

However, despite the excellent electrical conducting properties, an important drawback of PEDOT is its poor solubility in common organic and inorganic solvents: this implies that thin films cannot be obtained. Such an issue is only partially circumvented in PEDOT-Sulfonated Polystyrene (SPS) blends (PEDOT_SPS) [10].

In the present work, we have synthesized PEDOTs characterized by full solubility of the obtained products in the organic reaction solvents chosen, via a high-concentration solvent based emulsion polymerization of EDOT and ferric sulfate as oxidant species. The organic solvents tested are N, N-dimethylformamide (DMF), dimethylacetamide (DMAc), dimethyl sulfoxide (DMSO) and N-methyl-2-pyrrolidone (NMP).

In order to improve PEDOT conductive properties, the doping agents based on sulfonic groups commonly used with PEDOT are 2-Naphthalenesulfonic acid, para toluene sulfonic acid or others sulfonated organic molecules [11]. Besides these species, also Sulfonated Polyarylethersulfone (SPAES), obtained as reported in our previous work [12], can be used as doping agent thanks to both the charge separation present in SPAES structure deriving from the use of a pre-sulfonated comonomer and the possibility to modulate the moieties of sulfonic groups along the polymeric chains. In this work, SPAESs with different degree of sulfonation (DS), i.e. $0.5,0.75$ and $1.0\left(\mathrm{meq} \mathrm{SO}_{3}{ }^{-} \cdot \mathrm{g}^{-}\right.$ 1 of polymer), were synthesized via polycondensation reaction using a sulfonated comonomer, 2,5dihydroxybenzene-1-sulfonate potassium salt. The oxidative polymerization of EDOT with ferric sulfate as oxidant and SPAES as dopant (used at 1\% w/w respect to EDOT monomer) was performed.

It was found that PEDOT_SPAESs electrochemical properties are in general better than the ones obtained for not doped PEDOT, commercial PEDOT doped with 2-Naphthalenesulfonic acid (PEDOT_NaSA) and neat SPAES, and furthermore these properties increase as SPAESs DS increases. The chemical structure of PEDOT_SPAES samples was studied via wide-angle scattering (WAXS), comparing the not doped PEDOT and the commercial PEDOT doped with 2-Naphthalenesulfonic acid crystalline structures, with the amorphous structures obtained in the case of PEDOT_SPAESs polymers.

\section{EXPERIMENTAL}

3,4-Ethylenedioxythiophene (EDOT, >97\%), ferric sulfate $\left(\mathrm{Fe}_{2}\left(\mathrm{SO}_{4}\right)_{3} \cdot \mathrm{xH}_{2} \mathrm{O},>97 \%\right)$, acetone $(\geq 99.9 \%)$, Dimethylacetamide (DMAc, $\geq 99.5 \%)$, toluene $(99.8 \%$ anhydrous), Dimethylformamide (DMF, $\geq 99.8 \%)$, dimethylacetamide (DMAc, $\geq 99.5 \%$ ), dimethyl sulfoxide (DMSO, $\geq 99.5 \%$ ) and N-methyl-2-pyrrolidone (NMP, $\geq 99.5 \%$ ) were supplied by Sigma Aldrich and used without further purification.

Sulfonated Polyarylethersulfones (SPAES) with three different degree of sulfonation (DS) $-0.5,0.75$ and 1.0 (meq $\mathrm{SO}_{3} \cdot \mathrm{g}^{-1}$ of polymer) - were synthesized and characterized as reported in a previous work [12]. To synthesize PEDOT_SPAES samples, in a representative synthetic procedure performed in according with our previous work [13], a DMF based suspension of SPAES with $0.5 \mathrm{meq} \mathrm{SO}_{3}{ }^{-} \mathrm{g}^{-1}$ of polymer $(0.01 \mathrm{~g}$ in $5 \mathrm{~mL}$ DMF $)$ was mixed with EDOT $(1.00 \mathrm{~g})$ using a magnetic stirrer, at room temperature and under air atmosphere. A DMF based solution of ferric sulfate (1.64 $\mathrm{g}$ in $5 \mathrm{~mL} \mathrm{DMF})$ was added drop-wise to the suspension. The reaction mixture was stirred for 24 $\mathrm{h}$ at room temperature. The polymerization reaction was repeated changing both SPAES DS $-0.75,1\left(\mathrm{SO}_{3}^{-} \cdot \mathrm{g}^{-1}\right.$ of polymer) - and the solvent: DMAc, DMSO and NMP. The same procedure was adopted without SPAES to obtain not doped PEDOT samples and with 2-Naphthalenesulfonic acid to obtain EDOTs polymers doped with naphthalene based dopant.

Samples for WAXS analyses were prepared drying in a vacuum oven (around 4 mbar) at $50^{\circ} \mathrm{C}$ for $24 \mathrm{~h}, 20-25$ mg of polymeric solution. WAXS data were collected at the beamline ID15A at the ESRF synchrotron (Grenoble, France); sample flakes were loaded in $2 \mathrm{~mm}$-diameter borosilicate glass tubes and exposed 10 minutes to X-rays of wavelength $0.1771 \AA$; the detector used was a Pilatus CdTe 2M (Dectris); raw images were summed, background subtracted, and radially integrated using the libraries FabIO and PyFAI [14]. For electrochemical measurements, 20 $\mu \mathrm{L}$ of PEDOT_SPAES solutions were casted on the top of a glassy carbon electrode (GC). Prior to modification, the GC support electrode surface was initially cleaned with synthetic diamond powder (Aldrich, diameter $1 \mu \mathrm{m}$ ) on a Struers DP Nap wet cloth and rinsed in water. $0.1 \mathrm{M} \mathrm{KCl}$ aqueous solution was used as supporting electrolyte and $\mathrm{Ru}\left(\mathrm{NH}_{3}\right)_{6} \mathrm{Cl}_{3}$ was employed as model probe molecule. Electrochemical investigations were performed in a three electrodes cell with a saturated calomel reference electrode and a platinum wire counter electrode. An Autolab PGStat 30 (Ecochemie, The Netherlands) potentiostat/galvanostat equipped with a FRA module for impedance (EIS) measurements was used. GPES, FRA and Z-View programs were used for the EIS spectra and voltammetric analyses. 


\section{RESULTS and DISCUSSION}

A series of SPAESs with three different DS - 0.5, 0.75 and 1.0 (meq $\mathrm{SO}_{3}{ }^{-} \mathrm{g}^{-1}$ of polymer) - were successfully synthesized and used as PEDOT doping agents, thanks to the charge separation deriving from the sulfonated comonomer [12]. PEDOT_SPAESs were successfully synthesized via oxidative polymerization in four different organic solvents (DMF, DMAc, DMSO and NMP), according to our previous work [15], using ferric sulfate as oxidant species and SPAES with different DS as dopant (Fig. 1a). PEDOT_SPAESs are characterized by excellent solubility in the reaction solvents chosen for the polymerization procedures. Furthermore, the colour of the polymeric solutions obtained is dependent by the solvent reaction adopted (Fig. 1b), suggesting that the nature of the solvent used during the synthesis can affect the physico-chemical properties of the resulting polymers.
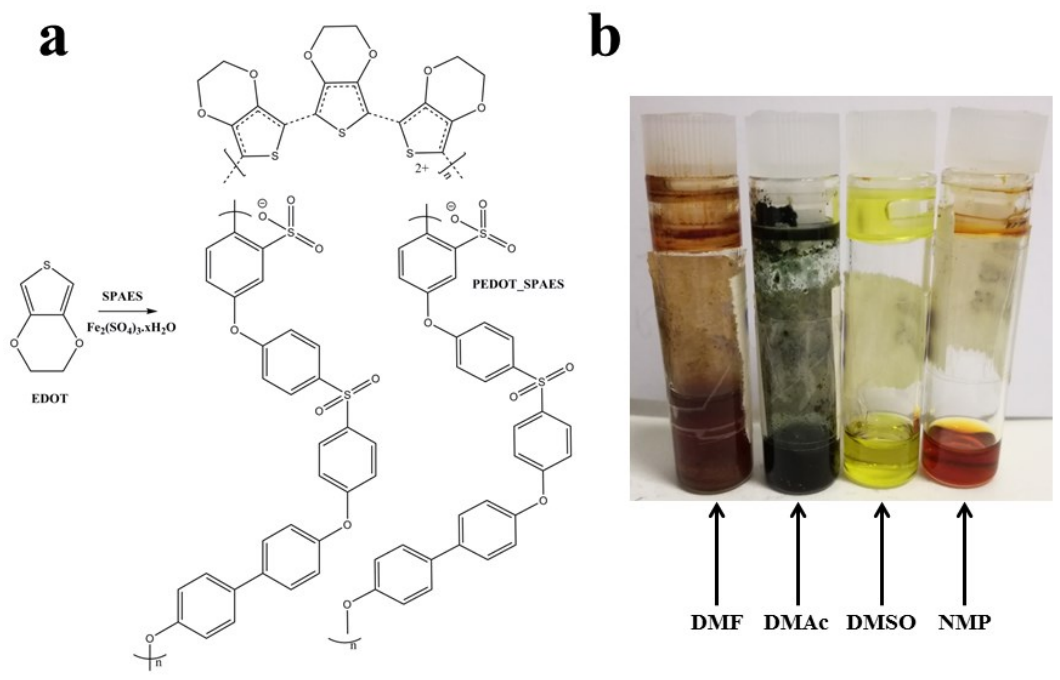

FIGURE 1. (a) PEDOT_SPAES synthetic route proposed; (b) PEDOT_SPAES solutions in DMF, DMAc, DMSO and NMP.

The chemical structure of PEDOT_SPAES polymers was studied via wide-angle scattering (WAXS), comparing undoped PEDOT and the commercial PEDOT doped with 2-Naphthalenesulfonic acid (PEDOT_NaSA) with the structures obtained in the case of PEDOT SPAESs using NMP as solvent (Fig. 2). It was found that PEDOT and PEDOT_NaSA are highly crystalline (Fig. $2 \mathrm{a}$ ), with very intense reflections at $q=0.5$ and $1.8 \AA^{-1}$ (corresponding to d-spacings of 12.57 and $3.49 \AA$, respectively). In PEDOT_SPAESs, no diffraction peaks are visible and the two broad low-q peaks tend to coalesce as the DS increases (Fig. 2b), showing that low SPAES loadings $(1 \% \mathrm{w} / \mathrm{w}$ respect to EDOT) are enough to disrupt the long-range ordered structure of PEDOT and produce amorphous blends. Studies of the PEDOT:SPAES interaction mechanism and the effect of other solvents on PEDOT_SPAES structure (i.e. DMF, DMAc and DMSO) are now in progress and will be presented in subsequent works.
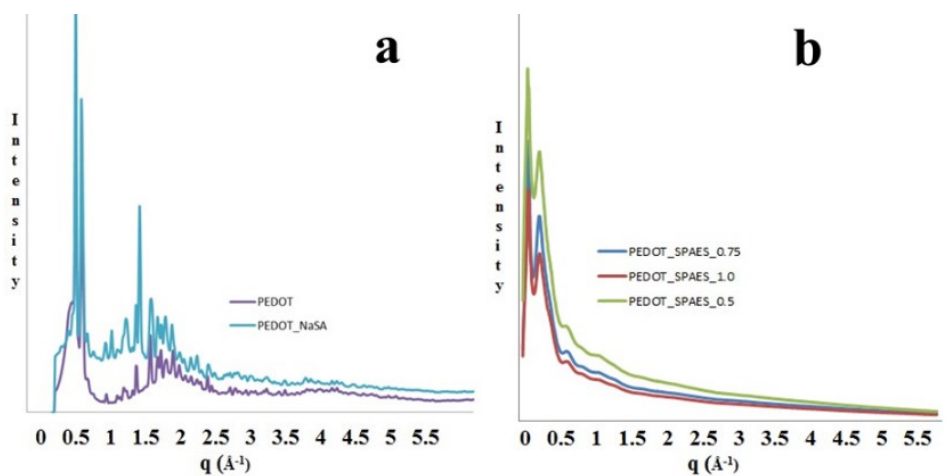

FIGURE 2. (a) WAXS spectra of PEDOT and PEDOT_NaSA; (b) WAXS spectra of PEDOT_SPAES with 0.5, 0.75 and 1.0 meq $\mathrm{SO}_{3}^{-\cdot} \cdot \mathrm{g}^{-1}$. 
PEDOT_SPAES polymeric solutions were casted onto the surface of a GC electrode and their electrochemical properties were compared with the ones of a neat GC and GCs modified with SPAES solutions. The cyclic voltammetries of SPAESs (Fig. 3a) were compared with the ones of PEDOT, PEDOT NaSA and PEDOT SPAESs (Fig. 3b) using NMP solvent for the preparation of the polymeric solutions $\left(30^{\circ} \overline{\mathrm{C}}, 250 \mathrm{mbar}\right)$; from the data obtained, it is worth of note the effect of both SPAES and SPAES DS on PEDOT electrochemical behavior, in terms of higher peak currents. The studies with DMF, DMAc and DMSO solvents and the variations of casting conditions are in progress and will be discussed in future works.

\section{a}

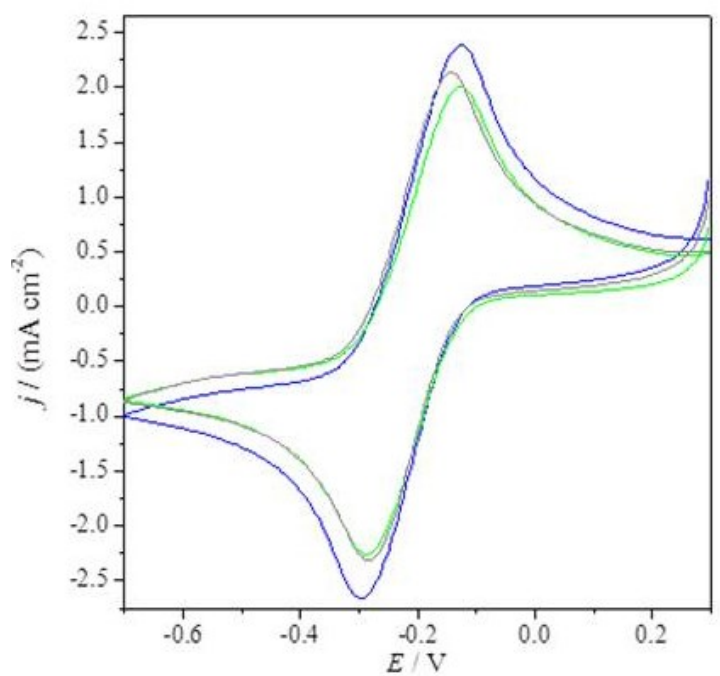

b

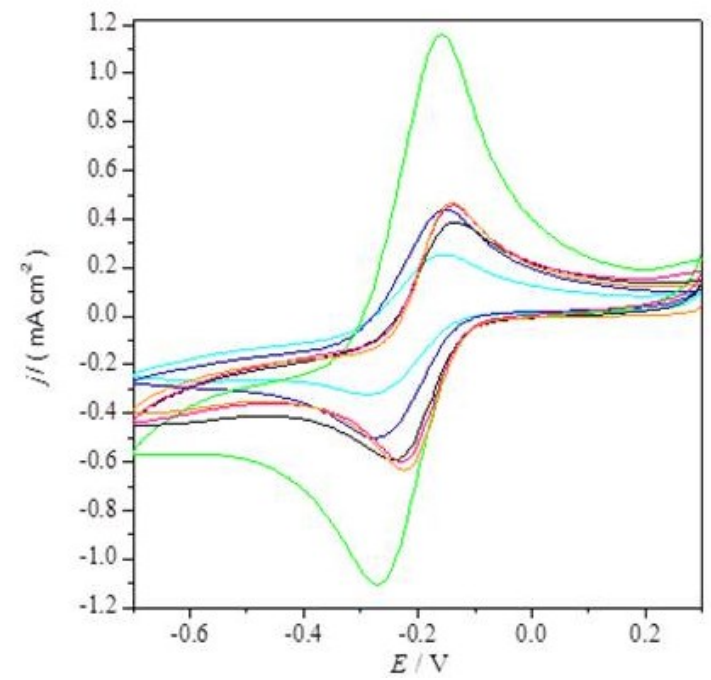

FIGURE 3. (a) Cyclic voltammetries of SPAES with 0.5 (grey line), 0.75 (green) and 1.0 (blue) meq $\mathrm{SO}_{3}{ }^{-\cdot} \cdot \mathrm{g}^{-1}$ of polymer; (b) Cyclic voltammetries of PEDOT (black), PEDOT_NaSA (pink), and PEDOT_SPAES with 0.5 (blue), 0.75 (light blue) and 1.0 (green) meq $\mathrm{SO}_{3}^{-} \cdot \mathrm{g}^{-1}$ of polymer.

\section{CONCLUSIONS}

A new synthesis of a high-concentration solvent-based emulsion polymerization of Poly(3,4Ethylenedioxythiophene) (PEDOT) doped with Sulfonated Polyarylethersulfone (SPAES), characterized by three increasing degree of sulfonation (DS), i.e. $0.5,0.75$ and 1.0 (meq $\mathrm{SO}_{3}{ }^{-} \cdot \mathrm{g}^{-1}$ of polymer), soluble in common organic solvents such as N, N-dimethylformamide (DMF), dimethylacetamide (DMAc), dimethyl sulfoxide (DMSO) and Nmethyl-2-pyrrolidone (NMP), was performed. It was found that PEDOT crystalline structure and cyclic voltammetries are significantly influenced by both SPAES and SPAES DS; to the authors' best knowledge, this is the first time that SPAESs were used as PEDOT dopant and that a study, though preliminary, related to SPAES effect on PEDOT properties was presented.

\section{REFERENCES}

1. Q. Xu, T. Song, W. Cui, Y. Liu, W. Lee, and B. Sun, ACS Appl. Mat. Inter 7, 3272-3279, (2015).

2. W. M. Sears, C. D. MacKinnon and T. M. Kraft, Synth. Met 161, 1566-1574, (2011).

3. J. Roncali, Chem. Rev 92, 711-738, (1992).

4. Z. Liu, K. Parvez, R. Li, R. Dong, X. Feng and K. Mullen, Adv. Mat 27, 669-675, (2015).

5. M. R. Lenz, N. M. Kroneneberg, F. Würthner and K. Meerholz, Org. Elec 21, 171-176, (2015).

6. M. Takayanagi, T. Gejo and I. Hanazaki, J. Phys. Chem 98, 12893-12898, (1994).

7. O. Pyshkina, A. Kubarkov and V. Sergeyev, Mat. Sci. Appl. Chem 21, 51-53, (2010).

8. Z. U. Khan, O. Bubnova, M. J. Jafari, R. Brooke, X. Liu, R. Gabrielsson and X. Crispin, J. Mat. Chem 3, 10616-10623, (2015).

9. Y. Lei, H. Oohata, S. Kuroda, S. Sasaki and T. Yamamoto, Synth. Met 149, 211-217 (2005).

10. D. Yoo, J. Kim, S. H. Lee, W. Cho, H. H. Choi, F. S. Kim and J. H. Kim, J. Mat. 11. J. E. McCarthy, C. A. Hanley, L. J. Brennan, V. G. Lambertini and Y. K. Gun'ko, J. Mat. 
12. V. Sabatini, S. Checchia, H. Farina, M. A. Ortenzi, Macromol. Res 483, 285-291 (2016).

13. V. Sabatini, H. Farina, M. A. Ortenzi, Polym. Eng. Sci. 57, 491-501 (2017).

14. E.B. Knudsen, H.O. Sørensen, J.P. Wright, G. Goret, and J. Kieffer, J. Appl Cryst., 46(2), 537-539 (2013) and J. Kieffer, and J.P. Wright, Powder Diffraction, 28(S2), S339-S350 (2013).

15. L. Falciola, S. Checchia, V. Pifferi, H. Farina, M.A. Ortenzi, V. Sabatini, Electrochim. Acta 194, 405-412 (2016). 